\title{
School-based Telehealth Programs: Integration and Process
}

\author{
Timothy D. Bruce
}

As a director of Telemedicine at LifeMD, identifying ways to integrate telehealth solutions into the arena of health and wellness practices and institutions is a constant challenge. Typically, two entities—business and government-are the beneficiaries of these services.

As an example, telemedicine clearly offers benefits within prison systems. Telemedicine lowers costs associated with transporting prisoners and improves access to healthcare specialists in correctional facilities. Hospital systems also benefit from telemedicine, as it allows for better coordination of patient care and facilitates patient monitoring remotely, thus reducing readmissions rates (among other benefits), according Lewis et al (Table 1) Whether the return on investment $(\mathrm{ROI})$ is a direct benefit in terms of increasing billing opportunities or for lowering operating costs, there are major financial incentives involved for these organizations to provide telemedicine services.

School-based telehealth programs straddle the line between government and private healthcare. Depending on the model the school follows, they can combine the "nonprofit" logistics of an educational institution with the variety and revenue-generating potential of "for profit" private medical practices. This area in telehealth has the potential to increase revenue and lower the costs associated with traditional office visits, while providing an important service to students in greatest need.

Two challenges to total acceptance and integration of school-wide telehealth initiatives are financial viability and educational barriers by groups participating in the program.

\section{Financial Viability}




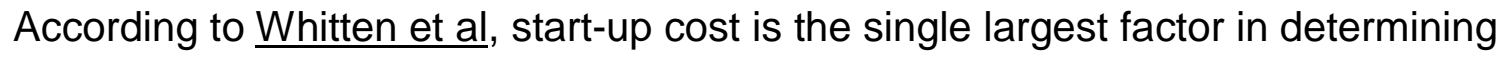
whether a district is capable of embarking on this type of project. A single telemedicine cart ranges from $\$ 20,000$ to $\$ 80,000$, and this may be prohibitive for a district needing to increase their ability to deliver additional healthcare benefits through telemedicine. However, when considered from the perspective of enhanced reach and service of school nurses and behavioral health specialists, the return on investment for a district can be significant.

For example, the cost of a machine suited for a school-based program is approximately $\$ 35,000$, with cost to the district of $\$ 7,000$ annually over 5 years. According to salary.com, the average school nurse salary in the United States is $\$ 45,985$ annually. The average salary of a certified medical assistant is $\$ 32,692$ annually-a difference of $\$ 13,293$ a year and $\$ 66,465$ over 5 years. Minus the cost of the machine, a single telemedicine cart can save a school district $\$ 31,465$ per machine over 5 years per location. By factoring in the potential cost savings associated with teachers using telemedicine consults in lieu of taking sick days to see a physician and the costs of a substitute teacher to take their place, it is possible to generate attractive numbers for a cash-strapped district.

With grants, as well as state and federal programs aimed at improving healthcare for students with chronic medical conditions (e.g., asthma, diabetes, and obesity), a school can potentially fund the startup fees at no additional cost to the district.

\section{Acceptance by School Nurses}

The school nurse serves a pivotal role to provide expertise and oversight of school health services, promotion of health education, and connection between the academic setting and healthcare settings. Yet, some school nurses feel threatened by the addition of a school-based telemedicine program. They view it as a threat to their jobs or an additional burden on an already full workload. For a telehealth program to be successful 
it is imperative to educate school nurses and medical assistants on the benefits to their careers and on their workload, as well as to the students and their families.

Nurses who engaged the telehealth program found it was a positive experience and beneficial to their responsibilities.

According to Mackert and Whitten, nurses who engaged the telehealth program found it was a positive experience and beneficial to their responsibilities. School nurses believed that having the ability to diagnose complex medical conditions and help treat students with chronic health conditions under the guidance of a licensed pediatrician, helped facilitate and empower them to provide a level of care that they previously were unable to provide on their own, either due to legal constraints or lack of certification.

School nurses who actually used telemedicine soon learned that they could provide superior care as measured by helping children feel better faster and getting students back in class where normally they required the students' parents to leave work and pick them up. According to McConnochie et al, $94 \%$ of parents surveyed about their telehealth experience said that the conditions treated via telehealth would have led to a doctor's visit or trip to an urgent care center or emergency department. And $91 \%$ stated that the school-based telehealth consultation allowed them to stay at work.

According to research by Young and Ireson, family savings from telehealth visits conducted at school ranged from $\$ 100$ to $\$ 225$ per encounter. The evidence suggests that school-based telehealth not only provides equal if not greater care, but it does so in a more cost-effective manner to schools and families alike. Additionally, telemedicine in urban and suburban childcare and elementary schools lightens family burdens.

\section{Pediatricians}


Local pediatricians may be concerned that school-based telehealth systems will appropriate their patients. To address this issue, the best strategy is to engage local physicians in taking part in the school-based initiative. When local physicians are busy and unable to connect with the school, the school clinic sends a request for a consultation, which may result in the student being seen by other providers within the network. There is also the possibility that the student will go without proper medical attention.

Programs are successful only when there is a total collaborative effort, with all parties agreeing on benefits for the recipients of the care. More over, local physicians must be convinced that they will be able to maintain their relationship with the community, increase patient visits through these consults, and expand their ability to provide of care and services. (Editor's note: Next month in TMT, Dr. Somesh Nigam discusses regulatory issues related to telemedicine and the patient-doctor relationship.)

\section{Conclusions}

Ultimately, the goal is to cost-effectively provide greater access to all students and close achievement gaps in order to improve the school culture and health. In addition, greater access must be a cost-effective solution for educational institutions facing increasing pressure to manage more students with medically complex requirements. Telehealth programs can have a positive impact on the students' lives as well as their families, schools, and community.

Long-term success requires flexibility at each stage of development and implementation.

Naturally school-based telehealth programs must be flexible. Rural districts and urban schools have varying needs that are as unique as the students themselves. Long-term success requires flexibility at each stage of development and implementation. 
Developing a streamlined system through coordinated efforts of multiple organizations collaboratively working towards the objective ensures that school telehealth programs will get up and running and then provide the desired results. As more schools demonstrate the success of their programs, they will become the vanguard of a new trend in school-based healthcare, providing an innovative way to leverage technology and make a positive impact on children's lives.

Tags: Acceptance, Bruce, business, cost cost-effective, financial, government, integration, LifeMD, nurse, pediatrician, pediatric, prison, process, school, viability

Timothy D. Bruce is Director of Telemedicine, Telehealth Services Department, www.LifeMD.com. (866) 954-8281 ext. 194.

Table 1. Reported benefits of telemonitoring used either alone or as part of a multidisciplinary care program for a variety of conditions.

- Reduce hospital bed-days occupancy

- High patient acceptance and compliance with telemonitoring

- Early detection of deterioration and reduce readmission rates and length of hospital stay in patients with heart failure

- Reduction in readmission charges

- Significant reduction in mortality at 6 months

- Reduction in the costs of hospital care, which offset the cost of video-consultation Source: Lewis et al 